\title{
DNA genetic artificial fish swarm constant modulus blind equalization algorithm and its application in medical image processing
}

\author{
Y.C. Guo ${ }^{1,2}$, H. Wang ${ }^{1}$ and B.L. Zhang ${ }^{1}$ \\ ${ }^{1}$ Jiangsu Key Laboratory of Meteorological Observation and Information \\ Processing, Nanjing University of Information Science \& Technology, \\ Nanjing, Jiangsu, China \\ ${ }_{2}^{2}$ Jiangsu Collaborative Innovation Center on Atmospheric Environment and \\ Equipment Technology, Nanjing, China \\ Corresponding author: Y.C. Guo \\ E-mail: guo-yecai@163.com
}

Genet. Mol. Res. 14 (4): 11806-11813 (2015)

Received January 28, 2015

Accepted May 15, 2015

Published October 2, 2015

DOI http://dx.doi.org/10.4238/2015.October.2.14

\begin{abstract}
This study proposes use of the DNA genetic artificial fish swarm constant modulus blind equalization algorithm (DNA-G-AFSCMBEA) to overcome the local convergence of the CMBEA. In this proposed algorithm, after the fusion of the fast convergence of the AFS algorithm and the global search capability of the DNA-G algorithm to drastically optimize the position vector of the artificial fish, the global optimal position vector is obtained and used as the initial optimal weight vector of the CMBEA. The result of application of this improved method in medical image processing demonstrates that the proposed algorithm outperforms the CMBEA and the AFS-CMBEA in removing the noise in a medical image and improving the peak signal to noise ratio.
\end{abstract}

Key words: Constant modulus blind equalization algorithm; Artificial fish swarm algorithm; DNA genetic algorithm;

Global optimal position vector 


\section{INTRODUCTION}

To eliminate inter-symbol interference and improve the performance of communication systems, some blind equalization algorithms have been used in the receiving terminal to compensate for or eliminate the inter-symbol interference of the channels. The constant modulus blind equalization algorithm (CMBEA) has the advantages of low complexity and strong robustness (Guo et al, 2012). However, the defects of this method include low convergence speed and local convergence.

The artificial fish swarm algorithm (AFSA) (Ma and Wang, 2009) is an optimization method based on individual autonomous animals. Although the AFSA has fast convergence speeds, it readily performs local searches. The DNA genetic algorithm (DNA-GA) (Rezaul Hasan, 2010) has the advantages of a genetic algorithm along with certain extra benefits such as global search functions and high search precision.

In this paper, a DNA genetic artificial fish swarm constant modulus algorithm (DNAG-AFS-CMBEA) is proposed via fusion of the AFSA with the DNA-GA. The experimental results confirm the validity of the proposed algorithm.

\section{METHODS}

\section{CMBEA}

Blind equalization is an algorithm-based method, which incorporates the statistical properties of the received signals; it contains no training sequences and can equalize the output and input signals to the greatest possible degree (Rao et al., 2011). In the CMEBA, we assume that $\boldsymbol{a}(k)$ denotes a zero mean independent identically distributed transmitted signal vector, $\boldsymbol{c}(k)$ denotes the impulse response vector of the channel, and $\boldsymbol{n}(k)$ denotes an additive white Gaussian noise vector, in addition to $y(k)$ representing the received signal vector to the equalizer. Furthermore, $\boldsymbol{f}(k)$ denotes the equalizer weight vector and $z(k)$ denotes the equalizer output signal. We give their relationships as follows:

$$
\begin{array}{cr}
\boldsymbol{y}(k)=\boldsymbol{c}^{\mathrm{T}}(k) \boldsymbol{a}(k)+\boldsymbol{n}(k) & \text { (Equation 1) } \\
z(k)=\boldsymbol{f}^{\mathrm{T}}(k) \boldsymbol{y}(k)=\boldsymbol{y}^{\mathrm{T}}(k) \boldsymbol{f}(k) & \text { (Equation 2) }
\end{array}
$$

where $T$ denotes the transposition operation. So, the error term of the CMBEA is defined as

$$
e(k)=|z(k)|^{2}-R^{2}
$$

where $R^{2}$ is the modulus value of the transmitted signals. In the CMBEA, the cost function is defined as

$$
J_{C M B E A}=E\left[e^{2}(k)\right] \quad \text { (Equation 4) }
$$

where $E$ denotes the mathematical expectation. The updated equation is written as 


$$
\boldsymbol{f}(k+1)=\boldsymbol{f}(k)-\mu e(k) z(k) \boldsymbol{y}^{*}(k)
$$

where $\mu$ is the step-size. The advantage of the CMBEA is that it is simple in structure and easy to implement. The drawbacks are the low convergence speed, the local convergence, and the large mean square error (MSE).

\section{DNA-GA-based AFSA}

The DNA-GA is an efficient algorithm with global search capabilities and fast convergence speeds. It is based on the reorganization of DNA molecules in monotonous form. The DNA-GA has the advantages of a traditional genetic algorithm. In addition, the DNA-GA is a good optimization method with flexible encoding and high search precision (Li and Lei, 2010).

In a permutation crossover operation, two individuals from the population are randomly selected as the parent bodies from the population. Two DNA sequences with the same length from each parent body can be selected and exchanged once. Two new individuals are obtained via replacement of the DNA sequences of the parent body.

In a translocation crossover operation, two individuals from the population are randomly selected as the parent bodies. A DNA sequence with the same length from each parent body is cut down. At the same time, a location from the remaining DNA sequence is selected to insert into the clipped DNA sequence.

In a mutation operator, an individual in the population is randomly selected as a parent body. The nucleobases with the highest frequency from parent body are replaced with the bases with the lowest frequency.

The purpose of the AFSA is to imitate fish swarm behaviors including preying, swarming, and following to achieve the optimal solution (Gao and Chen, 2010). The AFSA is simple and easy to implement. However, it also exhibits some defects such as local searching and slow convergence speeds.

Accordingly, we fused the DNA-GA with the AFSA. The resultant algorithm, DNAG-AFSA, is proposed to exhibit improved convergence speeds and strong robustness, and to accelerate the search for feasible solutions.

\section{DNA-G-AFS-CMBEA}

In order to further improve the performance of the CMBEA by increasing the convergence speed and reducing the mean square error, we introduced the DNA-G-AFSA into the CMBEA. The resultant algorithm, DNA-G-AFS- CMBEA, is therefore able to optimize the initial weight vector of the CMBEA using the functionality of the DNA-G-AFSA. is written as

The fitness function of the AFSA is defined by the cost function of the CMBEA and

$$
F\left(\boldsymbol{v}_{i}\right)=\frac{1}{J_{C M B E A}\left(\boldsymbol{f}_{i}\right)}=\frac{1}{E\left[e^{2}(k)\right]}
$$

where $J_{C M B E A}\left(f_{i}\right)$ denotes the cost function of the CMBEA. The optimization steps are given as follows: 
Step 1: Initialization of AFS. The initial population of AFS is written as $s=\left[s_{1}, s_{2}, \ldots\right.$, $\boldsymbol{s}_{\mathrm{N}}$, where $\boldsymbol{s}_{\mathrm{m}}$ is the initial position vector of the $m^{\text {th }}$ artificial fish and $N$ is the number of individuals.

Step 2: Calculation of fitness function. The fitness function of each artificial fish is calculated according to Equation (6). The maximum fitness function and its correspondence to the position vector are recorded in the bulletin board. The bulletin board is defined as the place to store the value.

Step 3: The preying, swarming, or following behavior of artificial fish. Each artificial fish carries out individual following, swarming, and preying behaviors. Accordingly, the current position vectors of the population are updated.

Step 4: DNA encoding. In order to obtain the DNA sequence of the position vector, the position vector of each artificial fish is encoded into the DNA sequence. When applied to the fish swarm in aggregate, these constitute the population of DNA sequences.

Step 5: Permutation crossover operation. Two DNA sequences from the population are randomly selected as the parent bodies. If a random number within 0 to 1 is less than the permutation crossover rate, the permutation crossover operation is carried out. The new population is obtained.

Step 6: Translocation crossover operation. Two DNA sequences are randomly selected as the parent bodies. If a random number within 0 to 1 is less than the translocation crossover rate, the translocation crossover operation is carried out. The new population is obtained.

Step 7: Mutation operator. A DNA sequence from the population is randomly selected as parent body and the mutation operator is applied. The new population is obtained.

Step 8: Coding, and updating the bulletin board. The position vectors of the artificial fish swarm are obtained from the population of DNA sequences via DNA decoding. The fitness functions of the artificial fish swarm are calculated. The maximum value is selected and compared with the original fitness function value preserved in the bulletin board. If the current fitness function maximum is greater than the original fitness function value preserved in the bulletin board, the current maximum and its corresponding position vector are substituted for the prior measures.

Step 9: Determination of the termination condition. The equivalence of the iteration to the maximum value is ascertained. If the condition does not hold, the iteration process goes back to step 3; otherwise, the record in the bulletin board is used as the initial optimization weight vector.

In order to validate the performance of the DNA-G-AFS-CMBEA, we carried out simulation tests. In the simulations, the transmitted signals were 16 QAM signals, the channel was $h=[0.9656-0.09060 .0578,0.2638]$, the signal to noise ratio was $20 \mathrm{~dB}$, the number of the equalizer weight coefficients was $L=16$, and the step-size was $\mu=0.00001$. The number of animals in the artificial fish swarm was $\mathrm{N}=16$, and the crowding factor was $\delta=0.618$. Figure 1 shows the simulated results. Figure 1A shows the MSE curves. Figure 1B shows the output constellations of the CMBEA. Figure 1C shows the output constellations of the AFSCMBEA. Figure 1D shows the output constellations of the DNA- G-AFS-CMBEA.

From Figure 1, we can see that the DNA-G-AFS-CMBEA outperforms the AFSCMBEA and CMBEA methods regarding improving the convergence speed and reducing the MSE. The MSE of the DNA-G- AFS-CMBEA is the smallest overall and its convergence speed is the fastest. Moreover, the output constellations of the DNA-G-AFS-CMBEA are more compact and clearer than are those of the CMBEA and they also have no phase rotation. However, the output constellations of the AFS-CMBEA exhibit the phase rotation phenomenon. 


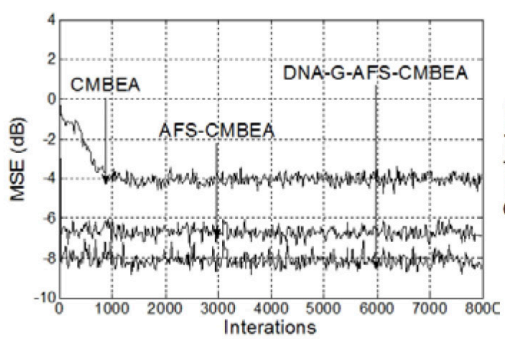

(A)

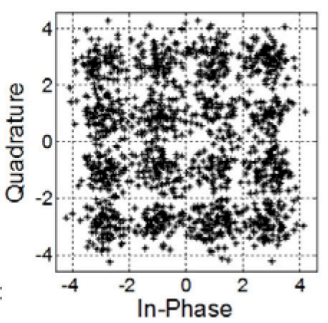

(B)

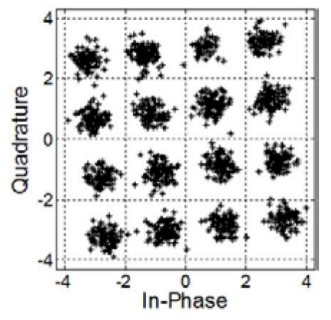

(C)

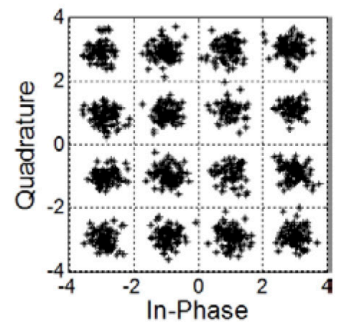

(D)

Figure 1. Simulated results of the various CMBEAs. A. MSE curves. B. CMBEA output constellations. C. AFSCMBEA output constellations. D. DNA-G-AFS-CMBEA output constellations. DNA-G-AFS-CMBEA, DNA genetic artificial fish swarm constant modulus blind equalization algorithm; MSE, mean square error.

\section{RESULTS AND DISCUSSION}

\section{Application in medical image processing}

In order to further validate the performance of the DNA-G-AFS- CMBEA, we employed this method to equalize a medical image. The noise of the medical image can be removed by the equalization of the noisy medical image. The steps of noisy medical image equalization using the DNA-G-AFS-CMBEA are given as follows: first, the two dimensional (2D) medical image is converted to a one dimensional (1D) image. The image dimension reduction model is shown in Figure 2.

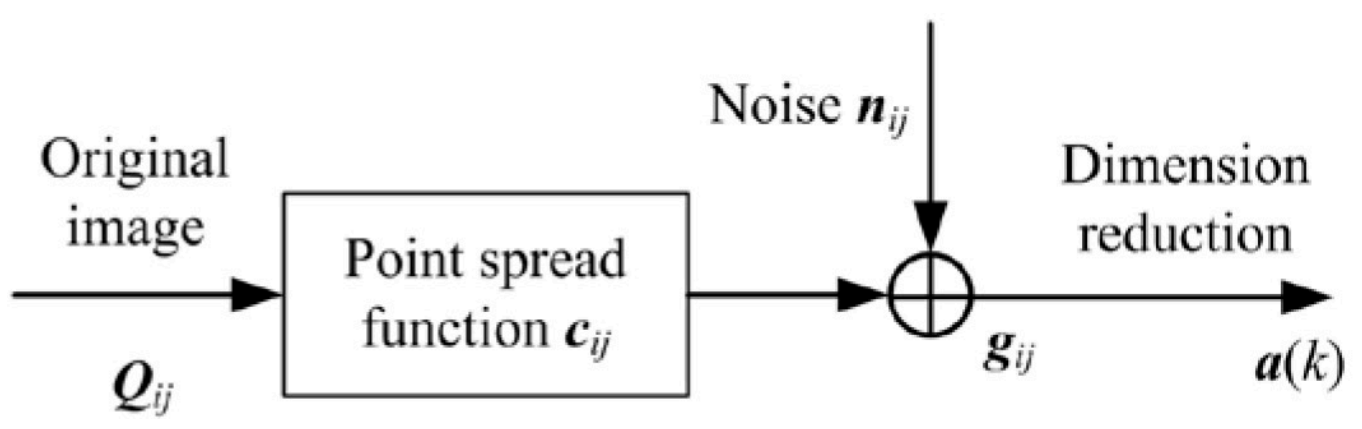

Figure 2. Medical image dimension reduction model. 
From Figure 2, $\boldsymbol{Q}_{i j}$ is defined as the original medical image; $\boldsymbol{g}_{i j}$ is a transformation of $\boldsymbol{Q}_{i j}$ using the point spread function $\boldsymbol{c}_{i j}$ with the different model supports $A$ x $A$; $\boldsymbol{n}_{i j}$ is additive white Gaussian noise with zero means and variance $\sigma_{n}^{2} . i, j=0,1, \ldots, M-1$. In order to use linear system theory, we show a high quality image system, a good approximation, and a linear translation invariant system (Zhang et al., 2011).

$$
\boldsymbol{g}_{i j}=\boldsymbol{Q}_{i j} \otimes \boldsymbol{c}_{i j}+\boldsymbol{n}_{i j}=\sum_{u=0}^{A-1} Q_{u, v} c_{i-u, j-v}+\boldsymbol{n}_{i j}
$$

where $\otimes$ denotes the convolution operation. In the dimension reduction processing of the original medical image, on the basis of the row transform method, the dimension reduction equation is written as

$$
\boldsymbol{a}(k)=\boldsymbol{g}_{i j}
$$

where $k=(i-1) M+j$ and $k \in\left[1, M^{2}\right] . M \times M$ is the size of the medical image. According to Equation (8), $\boldsymbol{a}(k)$ denotes the dimension reduction image of the noisy medical image and is regarded as the input signal to the blind equalizer. We then employed the DNA-G-AFSCMBEA for equalizing the input signal $\boldsymbol{a}(k)$ to the blind equalizer to obtain the output signal $z(k)$ to the blind equalizer. When the output signal $z(k)$ is obtained, the de-noised medical image $\mathbf{z}_{\mathrm{ij}}$ can be obtained on the basis of processing $z(k)$ according to the dimension increase method.

To evaluate the performance of the DNA-G-AFS-CMBEA in removing the noise in a medical image, the peak signal to noise ratio (PSNR) is selected as the evaluation index and defined as

$$
\operatorname{PSNR}=10 \lg \left(\frac{255^{2} \times M^{2}}{\sum_{i=1}^{M} \sum_{j=1}^{M}\left[z_{i j}-\boldsymbol{f}_{i j}\right]^{2}}\right)
$$

On the basis of the MATLAB environment and the DNA-G-AFS-CMBEA, simulation tests of medical image de-noising were carried out and compared with the results obtained using the AFS-CMBEA and the CMBEA. In the simulation, the point spread function was selected as a 10-by-10 Gaussian matrix with a mean of zero and a variance of 0.01 . The noise was regarded as Gaussian white noise with a mean of zero and a variance of 0.008 . The results of removing the noise in the medical image are shown in Figure 3 and Table 1.

From Figure 3 and Table 1, we can determine that the proposed DNA-G-AFS-CMBEA has a strong ability to remove the noise in a medical image; furthermore, its performance in improving PSNR is better than that obtained using the AFS-CMBEA and the CMBEA.

In order to apply the DNA-G-AFS algorithm to the CMBEA, we proposed a fused DNA-G-AFS-CMBEA method. The simulation results show that the MSE of the DNA-G-AFSCMBEA has the fastest convergence rate, the lowest MSE, and the best optimization. Furthermore, the DNA-G-AFS-DNA has more compact and clearer output constellations comparison with those of the AFS-CMBEA and the CMBEA. In this proposed algorithm, the DNA-GA and 
the AFSA are introduced into the CMBEA to further optimize the initial weight vector of the CMBEA. The simulation results show that the DNA-G-AFS-CMBEA method functions better than the AFS-CMBEA and the CMBEA in removing the noise in the medical image.

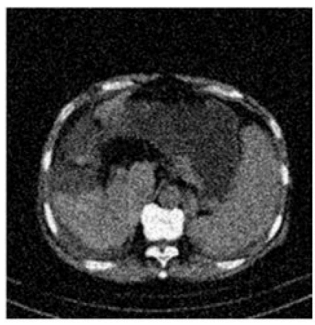

(A)

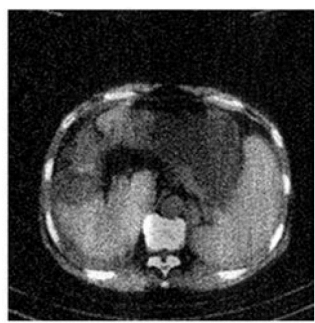

(B)

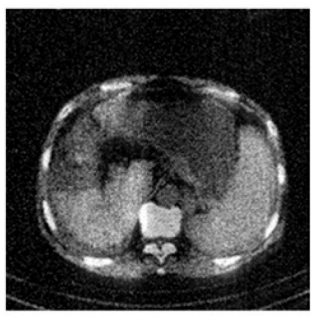

(C)

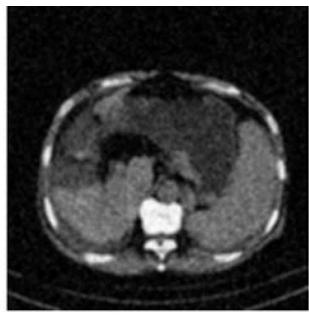

(D)

Figure 3. Application of the DNA-G-AFS-CMBEA to medical image processing for noise reduction. A) The noisy medical image. The results following CMBEA (B), AFS-CMBEA (C), and DNA-G-AFS-CMBEA (D) image processing. DNA genetic artificial fish swarm constant modulus blind equalization algorithm.

Table 1. Comparison of PSNRs between algorithms.

\begin{tabular}{lccc}
\hline Algorithm & DNA-G-AFS-CMBEA & AFS-CMBEA & CMBEA \\
\hline PSNR & 24.49 & 23.96 & 23.55 \\
\hline
\end{tabular}

PSNR = peak signal to noise ratio; DNA-G-AFS-CMBEA = DNA genetic artificial fish swarm constant modulus blind equalization algorithm.

\section{Conflicts of interest}

The authors declare no conflict of interest.

\section{ACKNOWLEDGMENTS}

Research supported by a major project of the University of Natural Science of Jiangsu Province (\#13KJA510001), a Jiangsu Scientific Research Achievements in Industrialization Project (\#JHB 2012-9), and a project funded by the Priority Academic Program Development of Jiangsu Higher Education Institutions (PAPD II), as well as Innovation Projects of Postgraduate Training in Jiangsu Province (\#SJZZ_0110, \#SJZZ_0111, and \#KYLX_0853).

\section{REFERENCES}

Gao YF and Chen YD (2010). The optimization of water utilization based on artificial fish-swarm algorithm. Sixth International Conference. Nat. Comput. 8: 4415-4419.

Guo YC, Hu LL and Ding R (2012). Orthogonal wavelet transform weighted multi-modulus blind equalization algorithm based on quantum particle swarm optimization. Acta Phys. Sin. 61: 281-287.

Li Y and Lei J (2010). A feasible solution to the beam-angle- optimization problem in radiotherapy planning with a DNAbased genetic algorithm. IEEE Trans. Biomed. Eng. 57: 499-508.

$\mathrm{Ma} \mathrm{H}$ and Wang YJ (2009). An artificial fish swarm algorithm based on chaos search. Fifth International Conference on Nat. Comput. 4: 118-121.

Rao W, Tan WQ, Li YM and Gao HJ (2011). New modified constant modulus algorithm for underwater acoustic communications. 2011 International Conference on Internet Computing and Information Services (ICICIS). Hong Kong, 563-566. 
Rezaul Hasan SM (2010). A novel mixed-signal integrated circuit model for DNA-protein regulatory genetic circuits and genetic state machines. IEEE Trans. Circ. Syst. I: Reg. Papers 55: 1185-1196.

Zhang LY, Sun YS and Zhang JY (2011). Medical CT image blind equalization algorithm based on orthogonal transform. J. Comput. Inform. Syst. 7: 3455-3461. 